\title{
Quality of Commercial Flavoured Oils and Seed Oils Using a Widespread Analytical Protocol
}

\author{
Luisa Mannina ${ }^{1,2}$, Stefania Cesa ${ }^{1}$, Riccarda Antiochia ${ }^{1}$, Silvia Vista ${ }^{1}$, Anatoly P. Sobolev ${ }^{2}$, Marcello Vitale ${ }^{3}$, \\ Maria Enrica Di Cocco ${ }^{4}$, Antonello Santini ${ }^{5} \&$ Antonio Casini ${ }^{1}$ \\ ${ }^{1}$ Dipartimento di Chimica e Tecnologie del Farmaco, Sapienza Università di Roma, Piazzale Aldo Moro 5, \\ I-00185 Rome, Italy \\ ${ }^{2}$ Istituto di Metodologie Chimiche, Laboratorio di Risonanza Magnetica “Annalaura Segre”, CNR, I-00015 \\ Monterotondo, Rome, Italy \\ ${ }^{3}$ Dipartimento di Biologia Ambientale, Sapienza Università di Roma, Piazzale Aldo Moro 5, I-00185 Rome, \\ Italy \\ ${ }^{4}$ Dipartimento di Chimica, Sapienza Università di Roma, Piazzale Aldo Moro 5, I-00185 Rome, Italy \\ ${ }^{5}$ Dipartimento di Farmacia, Università degli Studi di Napoli Federico II - Via Domenico Montesano, 49- 80131- \\ Naples, Italy \\ Correspondence: Luisa Mannina, Dipartimento di Chimica e Tecnologie del Farmaco, Sapienza Università di \\ Roma, Piazzale Aldo Moro 5, I-00185 Rome, Italy. E-mail: luisa.mannina@uniroma1.it
}

Received: June 10, 2013 Accepted: December 10, 2013 Online Published: April 23, 2014

doi:10.5539/jfr.v3n4p78 URL: http://dx.doi.org/10.5539/jfr.v3n4p78

\begin{abstract}
Commercial flavoured olive and olive-sunflower oils and seed oils with particular nutritional properties (e.g. linseed, safflower, sunflower, sesame and rice oils) were analysed using a widespread analytical protocol to have information on their quality and chemical composition. The protocol involved traditional determinations (free acidity, peroxide value, UV and VIS spectrophotometric indices, and fatty acid composition) along with ${ }^{1} \mathrm{H}$ and ${ }^{13} \mathrm{C}$ NMR analyses. Most of flavoured olive oils turned out to be lampante olive oils and not extra virgin as declared in the label on the bottle. In the case of olive-sunflower oils only a minor fraction of olive oil was revealed although these products are particularly expensive and the presence of olive oil is emphasized on the label. In some seed oils, refinement processes, not indicated on the bottle, were highlighted. Some compounds characteristic of specific seed oils were identified in the ${ }^{1} \mathrm{H}$ spectra.
\end{abstract}

Keywords: flavoured olive oil, seed oil, NMR, food quality

\section{Introduction}

Flavoured edible oils as well as seed oils constitute an interesting sector of vegetable oils market.

Production and consumption of flavoured olive oils has been growing for the last 15 years (Antoun \& Tsimidou, 1997) and market studies have demonstrated that consumers, especially from not Mediterranean areas, are interested in these products (Antoun \& Tsimidou, 1997). These oils are generically labelled as "dressing" since they are not subjected to the analytical controls required for olive oils (European Communities [EC], 2003 Regulation No 1989/2003) before their entries on the market. The price of these products is generally high although the lack of any control could induce the use of poor quality olive oils in the production. A few studies have focused on the stability of some flavoured olive oils (Antoun \& Tsimidou, 1997) and a preliminary NMR characterization of these products has been recently reported (Mannina et al., 2012).

Seed oils have been present for a long time in market as a low cost alternative to virgin olive oils. They are mostly produced by solvent extraction and therefore they have to be refined (Italian law, "Salari law", 27 January 1968- G.U. n.37 12/02/1968). Besides this type of seed oils, recently, a relatively small production of cold pressed seed oils, obtained in a way similar to virgin olive oils, has come out assuming some relevance.

Linseed oil mainly consists of triglycerides of essential $\alpha$-linolenic and linoleic fatty acids usually in a ratio of about $2: 1$, respectively. Therefore, consumption of linseed oil can be particularly useful to rebalance the ratio between $\omega-3$ and $\omega-6$ fatty acids. A suitable $\omega-3 / \omega-6$ ratio seems to be important to prevent either cardiovascular 
disease (Angerer \& von Schacky, 2000), or certain types of cancer (Dwivedi, Natarajan, \& Matthees, 2005; Thompson, Rickard, Orcheson, \& Seidl, 1996), and some inflammatory and neurodegenerative diseases.

Safflower oil has a very high content of linoleic acid (even more than $80 \%$ ) together with a low percentage of saturated fatty acids (5-13\%). Interestingly it contains CLA (conjugated linoleic acid), a popular supplement. It is referred both to have hypocholesterolemic properties and to induce weight loss (Silveira, Carraro, Monereo, \& Tébar, 2007; Wang \& Jones, 2004).

Sunflower oil is generally considered as a premium oil because of its light colour, mild flavour, high smoke point, high level of mono and diunsaturated fatty acids and low level of linolenic acid. Moreover, sunflower oil typically contains relatively high levels of lecithins, tocopherols, carotenoids and waxes (Warner, Vick, Kleingartner, Isaak, \& Doroff, 2003).

Sesame oil is rich in unsaturated fatty acids such as oleic and linoleic acids. The presence of a potent antioxidant $\gamma$-tocopherol prevents oxidation reactions. A characteristic and well represented component of sesame oil is sesamin (Rangkadilok et al., 2010) which belongs to the class of lignans.

Rice oil is usually refined being obtained by a solvent extraction technique. It has functional and nutritional properties (Sugano, Koba, \& Tsuji, 1999; Raghuram \& Rukimini, 1995) mainly due to the presence of $\gamma$-oryzanol and tocotrienols (Cabras \& Martelli, 2004; Belitz, Grosch, \& Schieberle, 2009).

An analytical method to control the chemical composition of commercial flavoured oils and seed oils can be useful to give consumers a concrete indication regarding the quality of purchased products. Therefore, in this paper, a widespread analytical protocol is applied to the analysis of commercial flavoured olive and olive-sunflower oils and seed oils (linseed, safflower, sunflower, sesame and rice oils) with particular nutritional properties. This protocol involves some conventional analyses required by EC rules for olive oils (free acidity, peroxide value, UV spectrometric indices and fatty acid composition) along with ${ }^{1} \mathrm{H}$ and ${ }^{13} \mathrm{C}$ NMR experiments extensively used in the edible oils characterization (Segre \& Mannina, 1997; Carsten, Raniero, \& Guillou, 2000; D'Imperio et al., 2010; Mannina \& Sobolev, 2011; Mannina, Sobolev, \& Segre, 2003; Mannina, Luchinat, Emanuele, \& Segre, 1999; Mannina et al., 2000).

\section{Material and Methods}

\subsection{Sampling}

Samples were purchased in supermarkets, groceries and organic food stores or were furnished directly by the producers (see Table 1). A detailed description, including the type of oil, mean price and info on the label of the bottles is also reported in Table 1. Note that a few samples present in the supermarket over the expiration date were also purchased and analyzed. 
Table 1. Description of edible oils analysed in this study

\begin{tabular}{|c|c|c|c|c|c|}
\hline $\begin{array}{l}\text { Sample } \\
\text { code* }\end{array}$ & Type of oil & $\begin{array}{c}\text { Price } \\
(€ / 1)\end{array}$ & $\begin{array}{l}\text { Expiration } \\
\text { date }\end{array}$ & Source & Label information \\
\hline $\mathrm{O} 1$ & basil & 9.80 & 28.02 .13 & $\mathrm{~S}$ & EVOO $99 \%$, dried basil 1\%, basil natural flavour. \\
\hline $\mathrm{O} 2$ & lemon & 15.96 & 20.12 .12 & $\mathrm{~S}$ & EVOO (97\%) with lemon ( $2 \%$ natural flavour spices). \\
\hline $\mathrm{O} 3$ & truffle & 16.76 & 14.12 .12 & $\mathrm{~S}$ & EVOO (99\%), truffle flavour $(1 \%)$ \\
\hline O4 & black truffle & 15.96 & 01.12 .12 & $\mathrm{~S}$ & EVOO (98\%) with black truffle (Tuber Aestivum and others of the group), flavour. \\
\hline O5 & black truffle & NA & 31.03 .14 & $\mathrm{P}$ & EVOO, black truffle 1\% (Tuber Aestivum Vitt) flavour. \\
\hline O6 & black truffle & 46.00 & 25.11 .12 & G & EVOO, flavours. \\
\hline O7 & white truffle & 46.40 & 31.08 .13 & G & $\begin{array}{l}\text { Italian EVOO, natural white truffle flavour }(1,5 \%) \text {, truffle flakes }(0,2 \%) \text {. TASTI } \\
\text { method. }\end{array}$ \\
\hline O8 & chilli pepper & 13.16 & 05.01 .13 & $\mathrm{~S}$ & EVOO $(97 \%)$, dried chilli pepper $(2 \%)$, chilli pepper natural flavour $(1 \%)$ \\
\hline O9 & chilli pepper & 11.96 & 06.13 & $\mathrm{~S}$ & EVOO, flavoured infusion of fresh chilli pepper. No dyes or preservatives. \\
\hline $\mathrm{O} 10$ & chilli pepper & 46.40 & 31.08 .13 & G & $\begin{array}{l}\text { Italian EVOO, dried chilli pepper }(1.5 \%) \text {, chilli pepper infusion ( } 1 \%) \text {. TASTI } \\
\text { method. }\end{array}$ \\
\hline $\mathrm{O} 11$ & chilli pepper & 15.96 & 20.12 .12 & $\mathrm{~S}$ & EVOO $(97 \%)$ with chilli pepper ( $2 \%$ natural flavour spices). \\
\hline $\mathrm{O} 12$ & chilli pepper & 9.80 & 22.02 .13 & $\mathrm{~S}$ & EVOO $97 \%$, dried chilli pepper $2 \%$, chilli pepper natural flavour $1 \%$. \\
\hline O13 & $\begin{array}{l}\text { garlic and chilli } \\
\text { pepper }\end{array}$ & NA & 02.09 .13 & $\mathrm{P}$ & $\begin{array}{l}\text { EVOO, garlic } 0.2 \% \text {, chilli pepper } 1 \% \text {, natural extracts infusion. Contains sulphur } \\
\text { dioxide. TASTI method. }\end{array}$ \\
\hline $\mathrm{O} 14$ & lemon and parsley & NA & 31.10 .13 & $\mathrm{P}$ & EVOO, lemon $1.5 \%$, parsley $0.5 \%$, natural extracts infusion. \\
\hline O15 & $\begin{array}{l}\text { juniper and } \\
\text { rosemary }\end{array}$ & NA & 31.03 .14 & $\mathrm{P}$ & EVOO, rosemary $1.5 \%$, juniper $1.5 \%$, natural extracts infusion. TASTI method. \\
\hline $\mathrm{OS} 1^{*}$ & basil & 30.62 & 16.12 .11 & $\mathrm{~S}$ & Sunflower oil, EVOO, basil 1\%, flavour. Cold infusion. \\
\hline OS2 & chilli pepper & 30.62 & 10.05 .13 & $\mathrm{~S}$ & Sunflower oil, EVOO, chilli pepper 3\%, flavour. Cold infusion. \\
\hline OS3 & chilli pepper & 30.62 & 18.03 .13 & $\mathrm{~S}$ & Sunflower oil, EVOO, chilli pepper 3\%, flavour. Cold infusion. \\
\hline OS4 & $\begin{array}{l}\text { truffle and } \\
\text { mushrooms }\end{array}$ & 30.62 & 08.07 .13 & $\mathrm{~S}$ & $\begin{array}{l}\text { Sunflower oil, EVOO, dried mushrooms } 0.5 \% \text {, truffle flavour, flavour. Cold } \\
\text { infusion. }\end{array}$ \\
\hline $\mathrm{OS}^{*}$ & $\begin{array}{l}\text { truffle and } \\
\text { mushrooms }\end{array}$ & 30.62 & 19.03 .12 & $\mathrm{~S}$ & $\begin{array}{l}\text { Sunflower oil, EVOO, dried mushrooms } 0.5 \% \text {, truffle flavour, flavour. Cold } \\
\text { infusion. }\end{array}$ \\
\hline OS6 & garlic and parsley & 30.62 & 31.03 .13 & $\mathrm{~S}$ & Sunflower oil, EVOO, flavour, garlic slices $0.7 \%$, parsley $0.5 \%$. Cold infusion. \\
\hline $\mathrm{OS} 7^{*}$ & garlic and parsley & 30.62 & 05.08 .11 & $\mathrm{~S}$ & Sunflower oil, EVOO, flavour, garlic slices $0.7 \%$, parsley $0.5 \%$. Cold infusion. \\
\hline S1 & linseed & NA & 01.01 .13 & $\mathrm{P}$ & Biological agriculture. Cold pressed without solvents. \\
\hline $\mathrm{S} 2$ & linseed & 51.90 & 01.01 .13 & $\mathrm{O}$ & Biological agriculture. Cold pressed without solvents. \\
\hline S3 & linseed & 15.68 & 30.09 .12 & $\mathrm{O}$ & Cold pressing. Swiss production. \\
\hline S4 & linseed & 17.20 & 01.12 .12 & $\mathrm{O}$ & $\begin{array}{l}\text { Contains } \omega-3 \alpha \text {-linolenic acid. Obtained only with physical processes: pressed } \\
\text { without solvents, then filtered. Biologic. }\end{array}$ \\
\hline S5 & sesame & 13.80 & 06.12 .12 & $\mathrm{O}$ & $\begin{array}{l}\text { Contains phospholipids. Obtained only with physical processes: pressed without } \\
\text { solvents, then filtered. Biologic. }\end{array}$ \\
\hline S6 & safflower & 10.80 & 07.12 .12 & $\mathrm{O}$ & $\begin{array}{l}\text { Natural source of vitamin K. Obtained only with physical processes: pressed } \\
\text { without solvents. Biologic. }\end{array}$ \\
\hline S7 & sunflower & NA & 01.05 .13 & $\mathrm{P}$ & Biological agriculture. Cold pressed without solvents. \\
\hline S8 & rice & 7.35 & 19.06 .13 & $\mathrm{~S}$ & Rice oil, vitamin E. \\
\hline
\end{tabular}

* $\mathrm{O}=$ flavoured Olive oils; OS = mixture of flavoured Olive and Sunflower oils; $\mathrm{S}=$ Seed oils.

${ }^{\circ} \mathrm{G}=$ grocery; $\mathrm{O}=$ organic food store; $\mathrm{P}=$ producer; $\mathrm{S}=$ supermarket.

$\mathrm{NA}=$ not available.

The symbol ${ }^{\star}$ denotes the samples present in the supermarket over the expiration date. 


\subsection{NMR Measurements}

NMR experiments were recorded at $300 \mathrm{~K}$ on a Bruker AVANCE 600 NMR spectrometer operating at the proton and carbon-13 frequencies of $600.13 \mathrm{MHz}$ and $150.9 \mathrm{MHz}$, respectively $\left(\mathrm{B}_{0}=14.1 \mathrm{~T}\right)$ and equipped with a Bruker multinuclear $Z$ gradient $5 \mathrm{~mm}$ probe head.

Samples for ${ }^{1} \mathrm{H}$ NMR analysis were prepared dissolving oil samples $(20 \mu \mathrm{L})$ in $\mathrm{CDCl}_{3}(700 \mu \mathrm{L})$ plus DMSO-d $\mathrm{d}_{6}$ $(20 \mu \mathrm{L})$, directly in the $5 \mathrm{~mm}$ NMR tube. The ${ }^{1} \mathrm{H}$ spectra were acquired using the following conditions: number of scans $1024, \pi / 2$ pulse $\sim 8 \mu \mathrm{s}$, time domain (TD) $64 \mathrm{~K}$ data points, relaxation delay plus acquisition time $3.5 \mathrm{~s}$ and spectral width $18.5 \mathrm{ppm} .{ }^{1} \mathrm{H}$ NMR spectra were obtained by the Fourier transformation of the Free Induction Decay (FID), applying an exponential multiplication with a line-broadening factor of $0.3 \mathrm{~Hz}$ and a zero filling $($ Size $=64 \mathrm{~K})$ procedure. ${ }^{1} \mathrm{H}$ NMR spectra were manually phased. Chemical shifts were reported with respect to the residual $\mathrm{CHCl}_{3}$ signal set to $7.26 \mathrm{ppm}$. The baseline was corrected using the Cubic Spline Baseline Correction routine in the Bruker TOPSPIN 1.3 software. The intensity of 1,3 and 1,2 diglycerides ${ }^{1} \mathrm{H}$ NMR signals at $3.99 \mathrm{ppm}$ and $3.64 \mathrm{ppm}$ respectively was measured using the semi-automatic peak picking routine of Bruker TOPSPIN software and normalized with respect to the resonance at $2.251 \mathrm{ppm}$, due to $\alpha$ - methylenic protons of all acyl chains, set to 1000 .

Samples for ${ }^{13} \mathrm{C}$ NMR analysis were prepared dissolving oil samples $(100 \mu \mathrm{L})$ in $\mathrm{CDCl}_{3}(600 \mu \mathrm{L})$ directly in the $5 \mathrm{~mm}$ NMR tube. ${ }^{13} \mathrm{C}$ NMR spectra were obtained using the following acquisition parameter: time domain $256 \mathrm{~K}$; spectral width $195 \mathrm{ppm}$; relaxation delay $2.5 \mathrm{~s}$. The GARP sequence was applied to perform proton decoupling. ${ }^{13} \mathrm{C}$ NMR spectra were obtained by the Fourier transformation of the FID, applying a Gaussian multiplication with a negative line-broadening factor of $-0.1 \mathrm{~Hz}$ and a Gaussian position of 0.2 and using a zero filling (Size = $256 \mathrm{~K}$ ) procedure. The resulting ${ }^{13} \mathrm{C}$ NMR spectrum was manually phased and the baseline was corrected automatically. Chemical shifts were reported with respect to the signal due to $\alpha$-methylenic protons of the glycerol moiety set to $62.36 \mathrm{ppm}$.

The assignment of the ${ }^{1} \mathrm{H}$ spectra was obtained by means of $2 \mathrm{D}$ experiments namely ${ }^{1} \mathrm{H}-{ }^{1} \mathrm{H}$ COSY (Correlation Spectroscopy), ${ }^{1} \mathrm{H}-{ }^{1} \mathrm{H}$ TOCSY (Total Correlation SpectroscopY), ${ }^{1} \mathrm{H}^{13}{ }^{13} \mathrm{C}$ HSQC (Heteronuclear Single Quantum Coherences) and ${ }^{1} \mathrm{H}^{13} \mathrm{C}$ HMBC (Heteronuclear Multiple Bond Coherences) (Braun, Kalinowski, \& Berger, 1998). All 2D experiments were carried out using 1024 data points in the $\mathrm{f} 2$ dimension and 512 data points in the f1 dimension. ${ }^{1} \mathrm{H}-{ }^{1} \mathrm{H}$ COSY experiments were processed in the magnitude mode with 512 x 512 data points. A recycle time of $2 \mathrm{~s}$ was used. The ${ }^{1} \mathrm{H}-{ }^{1} \mathrm{H}$ TOCSY experiments were recorded with a spin lock time of $80 \mathrm{~ms}$ and were processed in the phase sensitive mode with 512 x 512 data points. The delay for the evolution of long-range couplings in ${ }^{1} \mathrm{H}-{ }^{13} \mathrm{C}$ HMBC experiments was $80 \mathrm{~ms}$.

\subsection{Conventional Analyses}

Free acidity, peroxide value, UV spectrophotometric indices $\left(\mathrm{K}_{232}, \mathrm{~K}_{270}, \Delta \mathrm{K}\right)$ and fatty acid composition were evaluated according to the official methods described in EEC 2568/1991, EC 1989/2003, UNI EN ISO 660:2005, UNI EN ISO 3960:2007, UNI EN ISO 3656:2002 Regulations. For each sample all parameters were determined in triplicate. Free acidity was expressed as grams of oleic acid per $100 \mathrm{~g}$ of olive oil. Peroxide value was expressed in milliequivalents of active oxygen per kilogram of oil. Spectrophotometric analyses were performed according to the official method ISO 3656:2011 with a UV-Vis spectrophotometer Perkin Elmer Lambda 40. Gas-chromatographic fatty acid analyses were performed using a Perkin Elmer 8500 GC and a GC Master Dani (for seed oils) equipped with a capillary SP2380, $(60 \mathrm{~m}, 0.2 \mu \mathrm{m})$ column and a FID $\left(\mathrm{T}_{1}=140{ }^{\circ} \mathrm{C}\right.$, r.r. $4{ }^{\circ} \mathrm{C} / \mathrm{min}$, $\mathrm{T}_{2}=240{ }^{\circ} \mathrm{C}, \mathrm{T}_{\operatorname{det}} 250^{\circ} \mathrm{C}, \mathrm{T}_{\text {inj }} 250^{\circ} \mathrm{C}$ ).

Spectrophotometric analyses in the UV-visible region between 300 and $600 \mathrm{~nm}$ were performed on the seed oils and the flavoured mixture of olive and sunflower oils. The spectra were recorded in solution of $n$-hexane at $50 \%$ (v/v) with a spectrophotometer Perkin Elmer Lambda 40.

\section{Results and Discussion}

Results will be discussed according to the type of edible oil. All samples, namely, 15 flavoured olive oils, 7 flavoured olive- sunflower mixtures and 8 seed oils, were submitted to conventional analyses, namely, free acidity, peroxide value, fatty acid composition, and UV-spectrophotometric indices (not measured on seed oils) and NMR analyses. In the case of flavoured olive-sunflower oils and seed oils, Vis-spectrophotometric analyses were also performed. The results of these analyses are reported in Tables 2 and 3. 
Table 2. GC-FAME analysis of the oil samples (values are in percentage)

\begin{tabular}{|c|c|c|c|c|c|c|c|c|c|c|c|c|c|c|c|c|c|}
\hline Code & Type of oil & C14 & C16 & C16:1 & $\mathrm{C} 17$ & C17:1 & C18 & $\begin{array}{c}\text { C18:1 } \\
\text { tr }\end{array}$ & $\begin{array}{c}\text { C18:1 } \\
\text { cis }\end{array}$ & $\begin{array}{c}\text { C18:2 } \\
\text { tr }\end{array}$ & $\begin{array}{c}\text { C18:2 } \\
\text { cis }\end{array}$ & $\begin{array}{c}\text { C18:3 } \\
\text { tr }\end{array}$ & $\begin{array}{c}\text { C18:3 } \\
\text { cis }\end{array}$ & $\mathrm{C20}$ & C20:1 & $\mathrm{C22}$ & $\mathrm{C} 24$ \\
\hline 01 & basil & & 11.11 & 0.96 & 0.07 & 0.09 & 2.57 & & 78.22 & & 5.72 & & 0.59 & 0.37 & 0.26 & & \\
\hline $\mathbf{O 2}$ & lemon & & 13.45 & 0.76 & 0.11 & 0.16 & 2.72 & & 77.63 & & 4.29 & & 0.42 & 0.24 & 0.21 & & \\
\hline $\mathbf{O 3}$ & truffle & & 9.90 & 0.92 & 0.04 & 0.06 & 3.06 & & 80.42 & & 4.81 & & 0.44 & 0.20 & 0.15 & & \\
\hline O4 & black truffle & & 11.83 & 1.00 & 0.06 & 0.10 & 2.99 & & 75.39 & & 7.65 & & 0.48 & 0.31 & 0.19 & & \\
\hline O5 & black truffle & & 12.94 & 1.06 & 0.09 & 0.13 & 3.66 & & 75.17 & & 6.03 & & 0.56 & 0.35 & 0.19 & & \\
\hline O6 & black truffle & & 12.90 & 0.73 & & 0.10 & 3.16 & & 76.87 & & 5.13 & & 0.65 & 0.27 & 0.19 & & \\
\hline O7 & white truffle & & 16.62 & 1.82 & 0.17 & 0.28 & 1.77 & & 67.69 & & 10.30 & & 0.65 & 0.42 & 0.28 & & \\
\hline O8 & chilli pepper & & 10.35 & 0.87 & 0.06 & 0.21 & 3.06 & & 78.96 & & 5.20 & & 0.55 & 0.43 & 0.31 & & \\
\hline O9 & chilli pepper & & 11.91 & 0.96 & 0.07 & 0.14 & 2.60 & & 76.14 & & 6.96 & & 0.50 & 0.43 & 0.29 & & \\
\hline O10 & chilli pepper & & 18.41 & 2.10 & & 0.35 & 2.47 & & 67.10 & & 9.04 & & 0.53 & & & & \\
\hline 011 & chilli pepper & & 13.39 & 1.10 & 0.11 & 0.21 & 3.16 & & 76.26 & & 4.63 & & 0.52 & 0.41 & 0.21 & & \\
\hline 012 & chilli pepper & & 11.41 & 0.84 & 0.04 & 0.10 & 3.17 & & 78.28 & & 5.18 & & 0.51 & 0.30 & 0.17 & & \\
\hline 013 & garlic and chilli pepper & & 12.76 & 1.16 & 0.07 & 0.19 & 2.86 & & 72.84 & & 8.76 & & 0.58 & 0.46 & 0.32 & & \\
\hline 014 & lemon and parsley & & 12.83 & 1.16 & 0.03 & 0.10 & 2.99 & & 72.73 & & 8.93 & & 0.58 & 0.43 & 0.22 & & \\
\hline 015 & juniper and rosemary & & 14.05 & 1.72 & & 0.13 & 3.11 & & 71.54 & & 8.19 & & 0.55 & 0.49 & 0.22 & & \\
\hline OS1 ${ }^{*}$ & basil & 0.09 & 6.80 & 0.19 & 0.05 & 0.03 & 4.20 & & 30.52 & & 56.71 & & 0.08 & 0.26 & 0.19 & 0.67 & 0.21 \\
\hline OS2 & chilli pepper & & 8.43 & & & & 3.58 & & 28.11 & & 59.17 & & 0.37 & 0.20 & 0.14 & & \\
\hline OS3 & chilli pepper & & 8.91 & 0.25 & & 0.16 & 3.51 & & 28.42 & & 57.98 & & 0.50 & 0.12 & 0.15 & & \\
\hline OS4 & truffle and mushrooms & & 6.58 & 0.20 & & & 3.18 & & 35.25 & & 54.21 & & 0.24 & 0.17 & 0.17 & & \\
\hline $\mathrm{OS5}^{+}$ & truffle and mushrooms & 0.08 & 6.69 & 0.15 & 0.04 & 0.02 & 4.13 & & 28.76 & & 58.70 & & 0.07 & 0.26 & 0.18 & 0.69 & 0.22 \\
\hline OS6 & garlic and parsley & & 7.81 & 0.26 & 0.12 & 0.18 & 3.68 & & 32.95 & & 54.30 & & 0.51 & 0.13 & 0.06 & & \\
\hline $\mathrm{OS7}^{\star}$ & garlic and parsley & & 9.74 & 0.23 & & 0.14 & 3.92 & & 31.46 & & 53.41 & & 0.27 & 0.21 & 0.13 & & \\
\hline S1 & linseed & & 5.56 & 0.09 & 0.06 & 0.06 & 4.13 & 0.01 & 21.08 & 0.10 & 18.04 & 0.37 & 49.88 & 0.14 & 0.16 & 0.18 & 0.10 \\
\hline $\mathbf{S 2}$ & linseed & 0.05 & 5.67 & 0.08 & 0.06 & 0.04 & 3.56 & & 18.58 & 0.08 & 15.96 & 0.42 & 55.04 & 0.13 & 0.13 & 0.13 & 0.08 \\
\hline S3 & linseed & 0.04 & 5.66 & 0.08 & 0.06 & 0.04 & 4.57 & & 18.20 & 0.09 & 14.79 & 0.47 & 55.50 & 0.14 & 0.12 & 0.14 & 0.10 \\
\hline S4 & linseed & 0.05 & 6.12 & 0.10 & 0.06 & 0.04 & 4.57 & & 19.09 & 0.09 & 14.13 & 0.41 & 54.83 & 0.15 & 0.11 & 0.16 & 0.10 \\
\hline S5 & sesame & 0.02 & 10.10 & 0.14 & 0.07 & 0.04 & 5.14 & & 40.07 & 0.10 & 42.78 & & 0.52 & 0.59 & 0.18 & 0.15 & 0.10 \\
\hline S6 & safflower & 0.12 & 7.45 & 0.12 & 0.04 & 0.03 & 2.51 & 0.02 & 14.92 & & 73.32 & 0.01 & 0.35 & 0.43 & 0.19 & 0.32 & 0.18 \\
\hline S7 & sunflower & 0.08 & 6.24 & 0.09 & 0.06 & 0.04 & 3.80 & & 25.86 & & 62.50 & & 0.06 & 0.25 & 0.14 & 0.66 & 0.22 \\
\hline S8 & rice & 0.46 & 20.97 & 0.22 & 0.04 & & 1.88 & & 40.35 & 0.44 & 32.22 & 0.22 & 1.29 & 0.78 & 0.45 & 0.25 & 0.41 \\
\hline
\end{tabular}

The symbol denotes the samples present in the supermarket over the expiration date.

\subsection{Flavoured Olive Oils}

Flavoured olive oils (bottles of 125-250 mL) were purchased in supermarkets and groceries or furnished directly by producers. The price ranges from $9.80 € / \mathrm{L}$, in the case of basil (O1) and chilli pepper (O12) olive oils purchased in supermarkets, up to $46.40 € / \mathrm{L}$ in the case of white truffle $(\mathrm{O} 7)$ and chilli pepper (O10) samples purchased in groceries. All samples are declared to be extra virgin olive oils. Only in the case of two very expensive flavoured oils (O7 and O10) the geographical origin is reported, being labelled as Italian extra virgin olive oils.

Free acidity and peroxides values of these samples are distributed in a range from 0.2 to $0.6 \%$ and from 2.9 to 19.9 meq $\mathrm{O}_{2} / \mathrm{kg}$, respectively (see Table 3). These values are within the limits ( $\leq 0.8 \%$, and $\leq 20.0 \mathrm{meq} \mathrm{O}_{2} / \mathrm{kg}$ ) of "extra-virgin" quality category established by the EC Regulation 1989/2003. On the other hand, UV specific absorption values confirm the "extra-virgin" quality category only for white truffle sample (O7). In fact white 
truffle sample has the $\mathrm{K}_{270}$ absorbance of 0.19 whereas all the other oils have the $\mathrm{K}_{270}$ absorbance greater than the 0.22 limit (EC 1989/2003). In particular, three samples (O1, O12 and O14) can be defined as virgin oils having the $\mathrm{K}_{270}$ absorbance between 0.22 and 0.25 whereas the other samples can be defined as lampante with the $\mathrm{K}_{270}$ absorbance greater than 0.25 . According to $\mathrm{K}_{232}$ absorbance, twelve samples are within the limits established for extra virgin oils $(\leq 2.5)$ whereas $\mathrm{K}_{232}$ absorbance value of $\mathrm{O} 1$ sample is within the limit of virgin category (between 2.5 and 2.6) and $\mathrm{O} 10$ and $\mathrm{O} 13$ samples can be defined as lampante ( $\geq 2.6)$. The $\Delta \mathrm{K}$ values are all lower than the limit prescribed for extra virgin olive oils $(\leq 0.01)$.

The fatty acids GC profile is compatible with extra-virgin olive oil category (EC 1989/2003); only one chilly pepper (O10) sample shows the percentage of $\mathrm{C} 17: 1$ fatty acid slightly higher than the legal limit $(0.35 \%$ compared to $0.30 \%$ ).

Since the quality category of an oil is defined by the worst analytical result obtained in the analyses, only white truffle sample (O7) can be classified as "extra-virgin", O1, O12 and O14 samples can be classified as "virgin" whereas the other eleven oils are "lampante" according to the limits defined by EC Regulation 1989/2003.

This result was also confirmed by the measure of $s n-1,2$, and $s n-1,3$ diglycerides performed by means of ${ }^{1}$ H-NMR spectroscopy (Mannina, Patumi, Proietti, Bassi, \& Segre, 2001). This diglycerides measure can be considered an "indirect measure" of the olive oil acidity. The $s n-1,2$ diglycerides naturally present in olive oils are gradually transformed into $s n-1,3$ (Belitz et al., 2009) by an isomerisation process. Consequently over time the amount of $s n-1,2$ diglycerides decreases in the oil whereas the amount of $s n-1,3$ diglycerides increases (Pérez-Camino, Moreda, \& Cert, 2001; Spyros, Philippidis, \& Dais, 2004). The ratio between the intensities of $s n-1,2$, and $s n-1,3$ diglycerides NMR signals, (see Table 3), gives an approximate indication of olive oil age, quality and state of preservation. Extra virgin olive oils with the acidity between $0.4 \%$ and $0.8 \%$ have a $s n-1,2$, and $s n-1,3$ diglycerides ratio $>4$, whereas a $s n-1,2 / s n-1,3$ ratio $<4$ indicates a low quality, aged olive oil. In our case, only white truffle sample (O7) resulted to have a $s n-1,2, / s n-1,3$ diglycerides ratio greater than 4 . Note that this sample is the only one that conventional analyses classified as "extra virgin". 
Table 3. Conventional analyses and the ratio of the ${ }^{1} \mathrm{H}-\mathrm{NMR}$ normalised signal intensities $s n-1,2$ and $s n-1,3$ diglycerides

\begin{tabular}{|c|c|c|c|c|c|c|c|}
\hline Code & Type of oil & Acidity (\%) & Peroxide Value (meq $\mathrm{O}_{2} / \mathbf{k g}$ ) & $\mathbf{K}_{232}$ & $\mathbf{K}_{270}$ & $\Delta \mathbf{K}$ & Ratio $s n 1,2 / s n 1,3$ \\
\hline 01 & basil & 0.3 & 19.9 & 2.54 & 0.24 & -0.0015 & 2.30 \\
\hline 02 & lemon & 0.3 & 13.7 & 2.36 & 0.37 & 0.0075 & 2.57 \\
\hline 03 & truffle & 0.5 & 5.8 & 2.10 & 0.26 & 0.0050 & 2.54 \\
\hline 04 & black truffle & 0.3 & 7.2 & 1.78 & 0.26 & 0.0080 & 2.68 \\
\hline 05 & black truffle & 0.5 & 9.0 & 2.13 & 0.32 & 0.0100 & 2.04 \\
\hline 06 & black truffle & 0.6 & 2.9 & 2.43 & 0.31 & 0.0035 & 1.50 \\
\hline 07 & white truffle & 0.2 & 3.7 & 2.20 & 0.19 & -0.0040 & 4.50 \\
\hline 08 & chilli pepper & 0.5 & 13.1 & 2.14 & 0.29 & 0.0070 & 2.60 \\
\hline 09 & chilli pepper & 0.4 & 15.6 & 2.37 & 0.27 & 0.0055 & 2.85 \\
\hline 010 & chilli pepper & 0.3 & 17.9 & 2.94 & 0.35 & -0.0065 & 3.91 \\
\hline 011 & chilli pepper & 0.3 & 13.4 & 1.93 & 0.32 & 0.0045 & 2.20 \\
\hline 012 & chilli pepper & 0.5 & 18.5 & 2.20 & 0.23 & 0.0085 & 1.98 \\
\hline 013 & garlic and chilli pepper & 0.5 & 14.6 & 2.69 & 0.35 & -0.0015 & 2.11 \\
\hline 014 & lemon and parsley & 0.4 & 16.6 & 2.30 & 0.24 & 0.0030 & 1.94 \\
\hline 015 & juniper and rosemary & 0.5 & 12.5 & 2.47 & 0.32 & 0.0000 & 2.15 \\
\hline OS1* & basil & 0.2 & 27.2 & $>6$ & 3.51 & 0.5800 & 1.67 \\
\hline OS2 & chilli pepper & 0.2 & 15.8 & 4.33 & 3.41 & 0.2360 & 1.80 \\
\hline OS3 & chilli pepper & 0.2 & 12.7 & $>6$ & 3.25 & .2750 & 1.95 \\
\hline OS4 & truffle and mushrooms & 0.2 & 13.9 & 3.87 & 2.12 & 0.4730 & 1.77 \\
\hline OS5 $^{+}$ & truffle and mushrooms & 0.1 & 25.2 & $>6$ & 3.37 & 0.4680 & 1.78 \\
\hline OS6 & garlic and parsley & 0.2 & 15.2 & $>6$ & 3.45 & 0.4200 & 1.64 \\
\hline OS $7^{+}$ & garlic and parsley & 0.2 & 20.0 & $>6$ & 3.03 & 0.6790 & 1.71 \\
\hline S1 & linseed & 0.4 & 2.7 & & & & \\
\hline S2 & linseed & 1.8 & 8.0 & & & & \\
\hline S3 & linseed & 0.6 & 3.2 & & & & \\
\hline S4 & linseed & 3.2 & 2.4 & & & & \\
\hline S5 & sesame & 1.1 & 1.6 & & & & \\
\hline S6 & safflower & 0.9 & 7.8 & & & & \\
\hline S7 & sunflower & 0.3 & 3.4 & & & & \\
\hline S8 & rice & 0.3 & 2.3 & & & & \\
\hline
\end{tabular}

The symbol denotes the samples present in the supermarket over the expiration date.

These results suggest that, if we apply the quality criteria used for olive oils in the case of flavoured olive oils, samples, analyzed within the expiration date, turned out to be lampante olive oils and not extra virgin olive oil as reported in the label on the bottle. It is possible either that the bottles were badly stored or that olive oils of low quality were used for the production of these flavoured oil. Whatever the reason of this result, the important aspect is that consumers buy a product of poor quality at a very high price.

\subsection{Flavoured Olive-Sunflower Oils}

Some flavoured oils are mixtures of olive oil, sunflower oil and aromas, see Table 1. We decided to buy and analyze also a few samples left on the shelf of supermarket beyond the expiration date. Free acidity values of flavoured olive-sunflower oils turned out to be very low, 0.1 or $0.2 \%$ (see Table 3 ). The low acidity is probably due to the presence of a high amount of sunflower oil usually obtained by the conventional refining technique 
involving a neutralization step. All the $s n-1,2 / s n-1,3$ diglycerides ratios, determined by ${ }^{1} \mathrm{H}$ NMR methodology, turned out to be very low (see Table 3 ), suggesting low quality products.

The extremely high values of UV absorbances $\left(\mathrm{K}_{270}>2\right)$ and the GC profiles confirm the prevalence of sunflower oil in these oils. Moreover, the oleic and linoleic acids amounts are close to those found in sunflower oil (sample S7), suggesting that only a minor fraction of olive oil is present in these oils. Note that these products are particularly expensive and the presence of olive oil in the product is emphasized on the label without any quantitative indication.

Spectrophotometric analyses in the UV-visible region, between 300 and $600 \mathrm{~nm}$, were also performed. It is important to underline that extra-virgin olive oil samples show absorption bands in the visible area mainly due to carotenoids and chlorophyll. Samples OS2 and OS3 (chilli pepper) and OS6-OS7 (garlic and parsley) show distinct absorption bands in the visible region (see Figure 1). These bands are very low in sample OS1 (basil) and completely absent in samples OS4 and OS5 (truffle and mushrooms), see Figure 1, thus suggesting a refinement procedure.
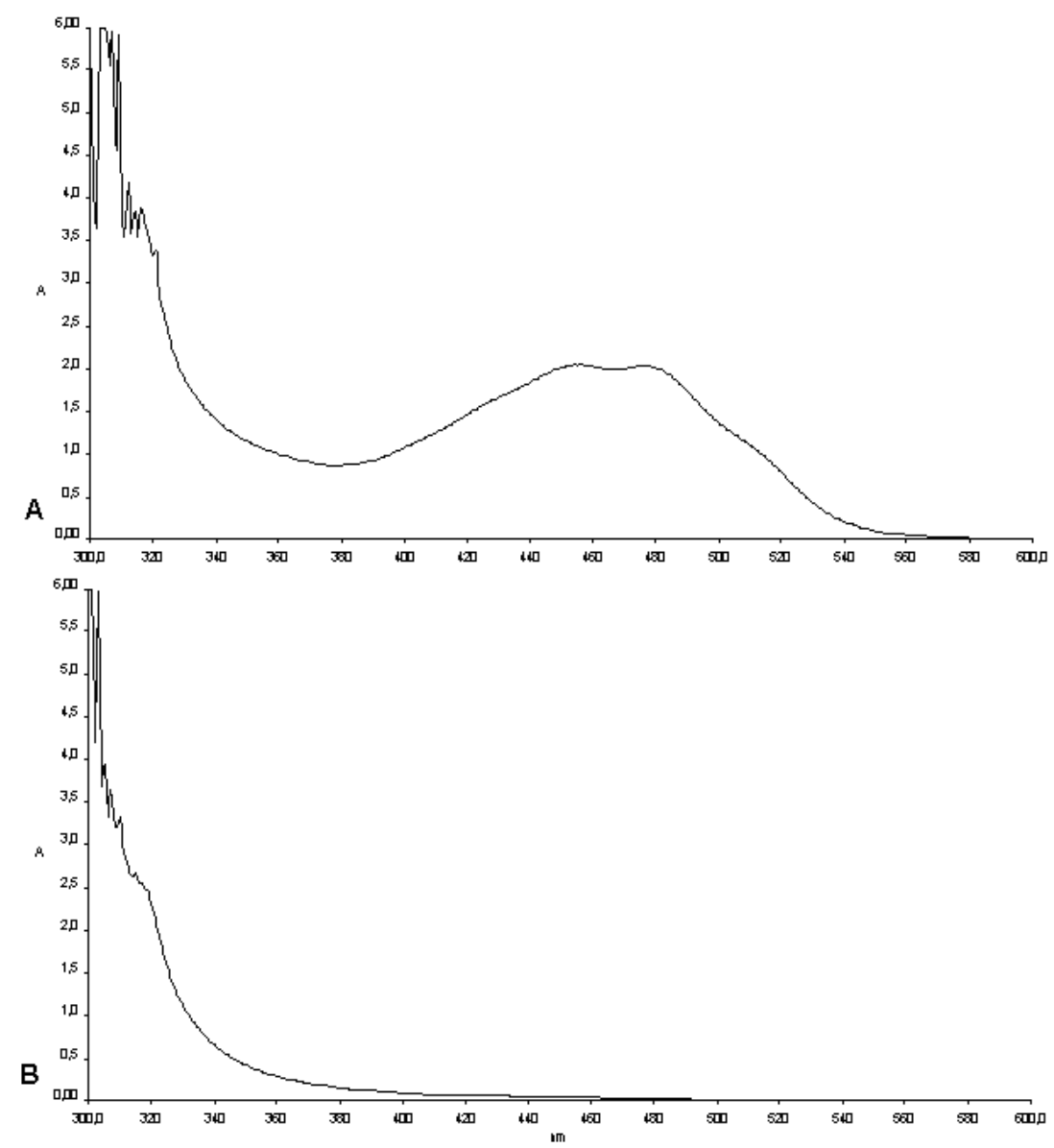

Figure 1. The UV-visible spectra in the region of $300-600 \mathrm{~nm}$ of (A) sample OS2 (chilli pepper) and (B) sample OS5 (truffle and mushrooms)

As expected, expired samples OS1, OS5 and OS7 showed high values of peroxide indices (see Table 3) due to oxidation reactions occurred over the time.

\subsection{Seed Oils Analyses}

The "family" of seed oils comprehends many vegetable oils with different chemical composition and properties. Some seed oils are produced by a cold pressing followed by washing with cold water. In the case of cold pressure oils, it is not allowed to correct the acidity value as instead prescribed by law in the case of refined seed 
oils. Seed oils are not subjected to the regulation required for olive oils. However, the UNI EN ISO rules give the maximum value of acidity $(0.5 \%)$ and peroxide value $\left(10.0 \mathrm{meq} \mathrm{O}_{2} / \mathrm{kg}\right)$ for refined oils. The UV spectrometric indices are not required. In the case of few cold pressed seed oils such as sunflower, soybean and maize seed oils, acidity and peroxide values upper limits are extended up to $2.0 \%$ and 15.0 meq $\mathrm{O}_{2} / \mathrm{kg}$, respectively.

Here, we have analyzed some seed oils with high nutritional value: namely linseed, safflower, sunflower, sesame and rice oils.

All samples, except rice oil, are produced, as declared in the label, by a cold pressing followed by washing with cold water. Free acidity values range from 0.3 to $3.2 \%$, (see Table 3 ). The acidity of these oils can depend on various factors, such as the seed moisture content, milling-time and manufacture techniques. Although it is not allowed to lower the acidity in cold pressed seed oils, the extremely low free acidity values of S1 and S3 linseed samples and S7 sunflower sample could suggest some neutralization treatment. Rice oil was certainly refined since it was obtained by an extraction process.

Peroxides indices are distributed in a restrict range from 1.6 to 8.0 meq $\mathrm{O}_{2} / \mathrm{kg}$ suggesting a good conservation. This is in agreement with the UNI EN ISO Italian rules which are applied to some of the investigated oils.

The GC fatty acids profile is specific for each type of seed oils. Linseed oils are rich in $\alpha$-linolenic acid whereas have a lower content of oleic, linoleic, and palmitic acids. Sesame and rice oils have the highest content of oleic acid together with a high content of palmitic and linoleic acids. Safflower and sunflower oils have the highest values of linoleic acid. These results are in agreement with literature data (Bondioli, Bernardi, Mariani Costantini, Sala, \& Venturini, 1999; Belitz et al., 2009).

It must be noted that although GC methods give the full composition of fatty chains, no information is given about the fatty chains distribution on glycerol ( $s n 1,3$ vs $s n 2$ in triglycerides). The acyl distribution on the glycerol moiety could be of crucial importance in the prevention of fraudulent addition of chemically esterified oils to seed oils. In fact, the random distribution of fatty acid chains on the glycerol moiety is a clear indicator of chemical esterification, whereas in seed oils saturated fatty acids occupy only sn 1,3 positions (Vlahov, 2009) and distribution of unsaturated fatty acids is not random. To obtain the valuable information about the acyl distribution on glycerol in analyzed seed oils ${ }^{13} \mathrm{C}$ NMR technique has been applied. In particular, the well resolved ${ }^{13} \mathrm{C}$ resonances of fatty acid carboxylic groups esterified in $s n 1,3$ positions are downfield shifted (ca 0.4 $\mathrm{ppm}$ ) with respect to the corresponding resonances of fatty acids esterified in the $s n 2$ position (see Figure 2 ). The ${ }^{13} \mathrm{C}$ resonances of carboxyl groups observed in the ${ }^{13} \mathrm{C}$ NMR spectra were the following: $173.50 \mathrm{ppm}$ (saturated fatty chains, $s n 1,3), 173.46 \mathrm{ppm}$ (oleic fatty chains, $s n 1,3), 173.45 \mathrm{ppm}$ (linoleic fatty chains, $s n 1,3), 173.44$ (linolenic fatty chains, $s n 1,3$ ), $173.06 \mathrm{ppm}$ (oleic fatty chains, $s n 2$ ), $173.05 \mathrm{ppm}$ (linoleic fatty chains, $s n 2$ ), 173.04 (linolenic fatty chains, $s n 2$ ). The ${ }^{13} \mathrm{C}$ signal of saturated fatty acids esterified in the $s n 2$ position of glycerol was not detected indicating the absence of chemically esterified triglycerides in all analyzed seed oils. The relative intensity of ${ }^{13} \mathrm{C}$ signals belonging to linoleic and linolenic acids esterified in $s n 1,3$ and $s n 2$ positions, (see Figure 2), suggests that these polyunsaturated fatty chains preferentially occupy $s n 2$ position, especially in rice and sesame oils. 

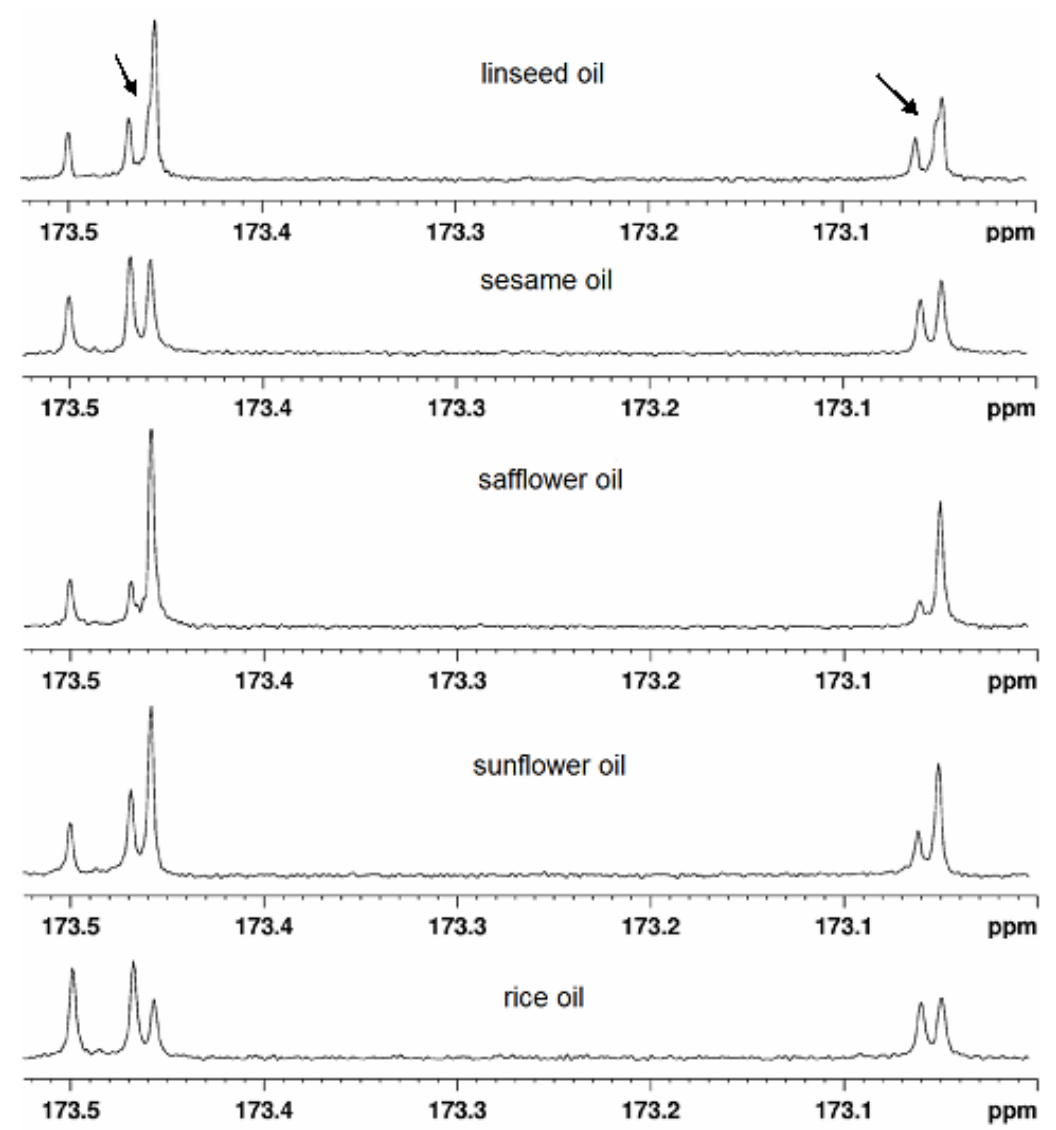

Figure 2. Expansion (173.5-173.0 ppm) of ${ }^{13} \mathrm{C}$ NMR spectra of seed oils. The arrows indicate partially overlapped signals of linoleic and linolenic acids carboxyl groups

Cold pressed seed oils do not require decolorization treatments since they are not submitted to refinement processes. On the other hand, decolorization treatment is required compulsorily in the case of refined seed oils and it is carried out by a diatomaceous earth or bentonite. Decolorization treatments can be verified using spectrophotometric analysis in the visible region. The absence of absorption bands in the VIS spectrum of a seed oil testifies the occurrence of decolorization.

Seed oils obtained simply by squeezing or treatments similar to those used for extra virgin olive oil, should have the absorption bands in the visible region. Consequently, if a cold pressed seed oil does not present these absorption bands it has surely been submitted to some refinement process.

In the case of investigated seed oils, safflower oil (S6) and all linseed oils (S1-S4) show the presence of absorption bands in the visible region whereas sesame, sunflower and rice oils do not show these absorptions. Therefore, probably, at least in sesame, sunflower and rice oils, a decolorizing treatment was applied.

Sterols are good indicators of the type of oils. The relative composition of the sterolic fraction can be regarded as a specific "fingerprint" of the particular seed oil. The ${ }^{1} \mathrm{H}$ NMR spectrum of edible oils shows in the 0.6-0.7 ppm spectral region the resonances of $\mathrm{CH}_{3}$ in position 18 of sterols (see Figure 3). In order to gain information about the sterols composition in any given vegetable oil it is possible to observe this useful small spectral region where no resonance of major compounds is present. 

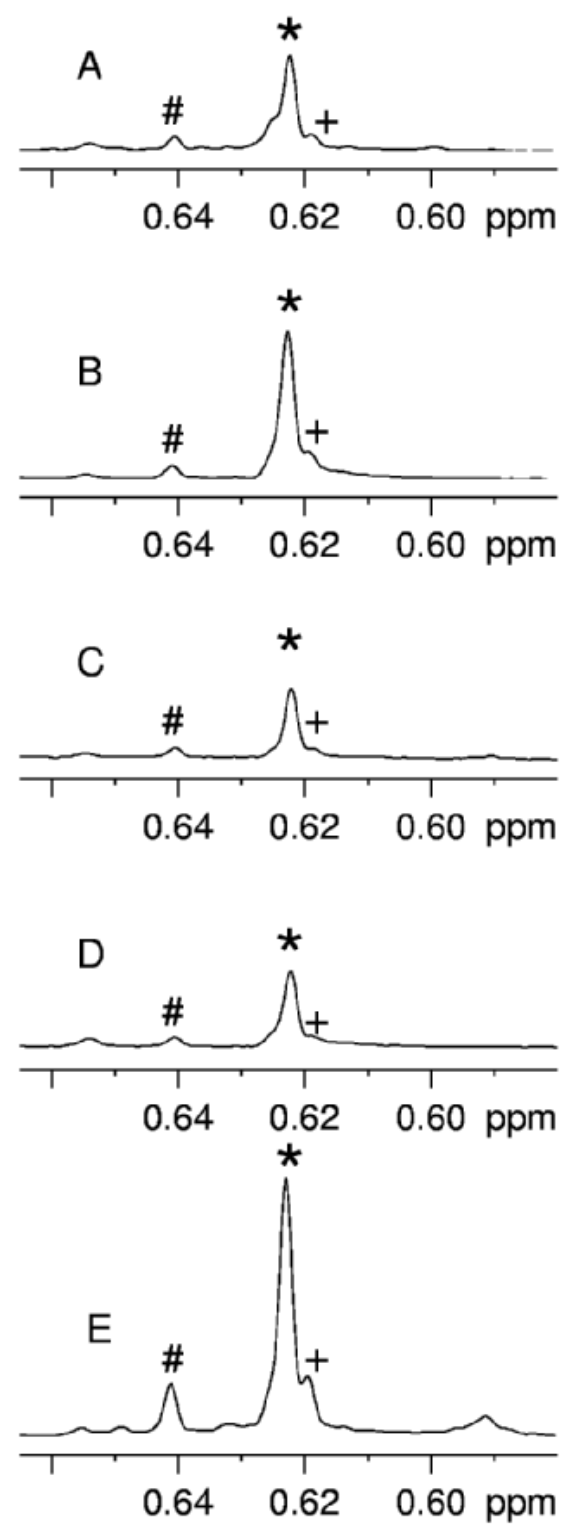

Figure 3. ${ }^{1} \mathrm{H}$ NMR spectral region $(0.66-0.58 \mathrm{ppm})$ of linseed oil S2 (A), sesame oil (B), safflower oil (C), sunflower oil (D), and rice oil (E). The symbols *, \#, + denote the signals of $18-\mathrm{CH}_{3}$ group of $\beta$-sitosterol, stigmasterol, and campesterol, respectively

Therefore, this spectral region can be read exactly as a chromatogram since the integral of each line is proportional to the concentration of the corresponding sterol. The sterol assignment was performed using literature data (Segre \& Mannina, 1997) and the addition of standards. $\beta$-Sitosterol, stigmasterol and campesterol were identified in all seed oils.

Besides the compounds present in all seed oils, several characteristic components were assigned for the first time in the ${ }^{1} \mathrm{H}$ NMR spectra of sesame and rice oils using $2 \mathrm{D}$ experiments. In the case of sesame oil the signals of sesamin and sesamolin, characteristic compounds of sesame seed oil (Rangkadilok et al., 2010), were assigned (see Table 4 and Figure 4). The assignment was consistent with the literature data for sesamin, characterized by NMR in several plant root extracts (Laggoune et al., 2011; Greger \& Hofer, 1980), and for sesamolin, characterized in sesame seeds (Kang, J. S. Kim, Jung, \& Y. H. Kim, 1995). 

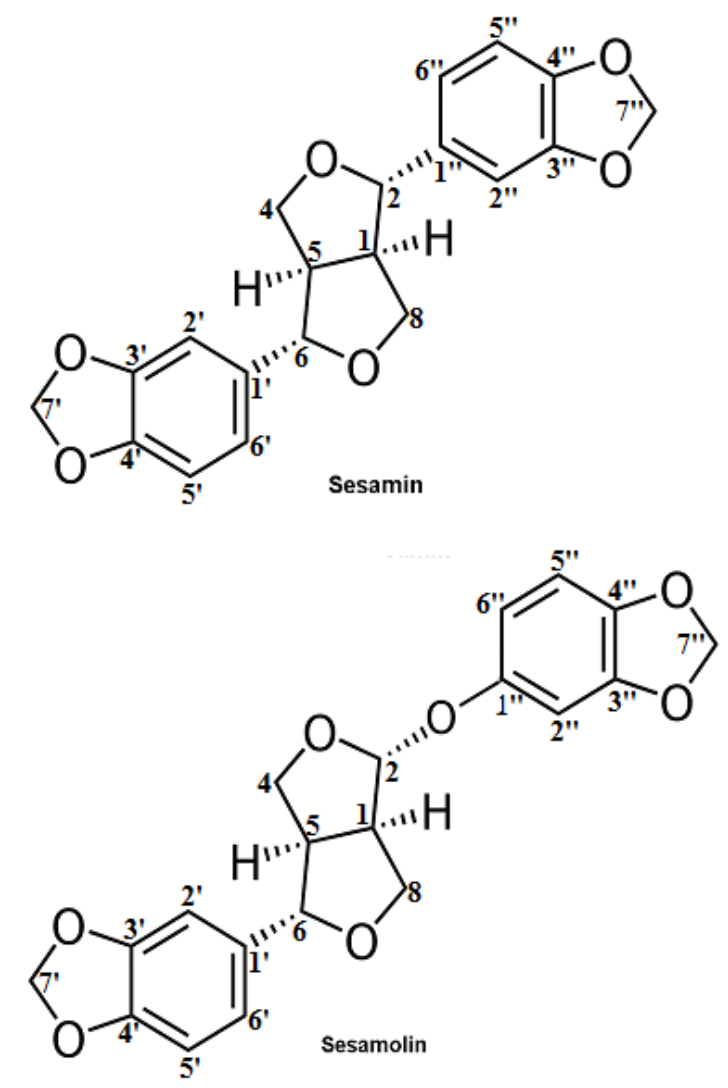

Figure 4. Structures of sesamin and sesamolin

Table 4. Assignments of the ${ }^{1} \mathrm{H}$ and ${ }^{13} \mathrm{C}$ NMR signals of sesamin and sesamolin in the sample of sesame oil. For the chemical structures see Figure 4

\begin{tabular}{lllll}
\hline \multicolumn{1}{c}{ Sesamin } & & \multicolumn{2}{c}{ Sesamolin } \\
Position & $\boldsymbol{\delta}_{\mathbf{H}}$ & $\boldsymbol{\delta}_{\mathbf{C}}$ & $\boldsymbol{\delta}_{\mathbf{H}}$ & $\boldsymbol{\delta}_{\mathbf{C}}$ \\
\hline C-1 & $2.99(\mathrm{~m})$ & 54.1 & 2.97 & 53.9 \\
\hline C-2 & $4.65(\mathrm{~d}, 4.4)$ & 85.7 & $5.44(\mathrm{~s})$ & 106.9 \\
C-4 & $3.81(\mathrm{dd}, 9.3,3.7)$ & 71.6 & 4.08 & \\
& $4.18(\mathrm{dd}, 9.3,6.8)$ & & $3.90(\mathrm{~d}, 9.0)$ & \\
C-5 & $2.99(\mathrm{~m})$ & 54.1 & 2.89 & 52.7 \\
C-6 & $4.65(\mathrm{~d}, 4.4)$ & 85.7 & $4.34(\mathrm{~s})$ & 87.1 \\
C-8 & $3.81(\mathrm{dd}, 9.3,3.7)$ & 71.6 & 4.38 & 71.0 \\
& $4.18(\mathrm{dd}, 9.3,6.8)$ & & 3.58 & 106.4 \\
C-2 & & 106.4 & $6.81(\mathrm{~d}, 1.6)$ & 108.1 \\
C-5 & $6.78(\mathrm{~d}, 1.7)$ & 108.0 & $6.72(\mathrm{~d}, 7.8)$ & 119.3 \\
C-6 & $6.72(\mathrm{~d}, 8.0)$ & 119.2 & $6.76(\mathrm{dd}, 7.8,1.6)$ & 101.1 \\
C-7 & $6.73(\mathrm{dd}, 8.0,1.7)$ & 100.8 & $5.86(\mathrm{~s})$ & 100.1 \\
C-2" & $5.89(\mathrm{~s})$ & 106.4 & $6.56(\mathrm{~d}, 2.5)$ & 107.8 \\
C-5" & $6.78(\mathrm{~d}, 1.7)$ & 108.0 & $6.64(\mathrm{~d}, 8.5)$ & 108.7 \\
C-6" & $6.72(\mathrm{~d}, 8.0)$ & 119.2 & $6.43(\mathrm{dd}, 8.5,2.5)$ & 100.9 \\
C-7” & $6.73(\mathrm{dd}, 8.0,1.7)$ & 100.8 & $5.90(\mathrm{~s})$ & \\
\hline & $5.89(\mathrm{~s})$ & & \\
\hline
\end{tabular}


A pattern of four ${ }^{1} \mathrm{H}$ NMR signals at 7.53, 6.98, 6.84, and $6.23 \mathrm{ppm}$ was observed in the ${ }^{1} \mathrm{H}$ NMR spectrum of rice oil. ${ }^{1} \mathrm{H}-{ }^{1} \mathrm{H}$ TOCSY and ${ }^{1} \mathrm{H}-{ }^{13} \mathrm{C}$ HSQC NMR experiments allow us to assign these signals to a $p$-cumaric acid derivative. Signals at 7.53 and $6.23 \mathrm{ppm}$ can be assigned to $\mathrm{CH}$ groups of trans double bond $\left(J_{H H}=16.0 \mathrm{~Hz}\right)$, whereas signals at 6.98 and $6.84 \mathrm{ppm}$ belong to ortho aromatic protons, as shown in Figure 5 (A). The subsistent in the carbonyl group denoted as $\mathrm{R}$ on the Figure 5 (A) remains unidentified. In any case it cannot be the unsubstituted $p$-cumaric acid as confirmed by the results of extraction in aqueous phase. In fact, no signal of unsubstituted $p$-cumaric acid was observed in the ${ }^{1} \mathrm{H}$ spectrum of the aqueous phase after the extraction.

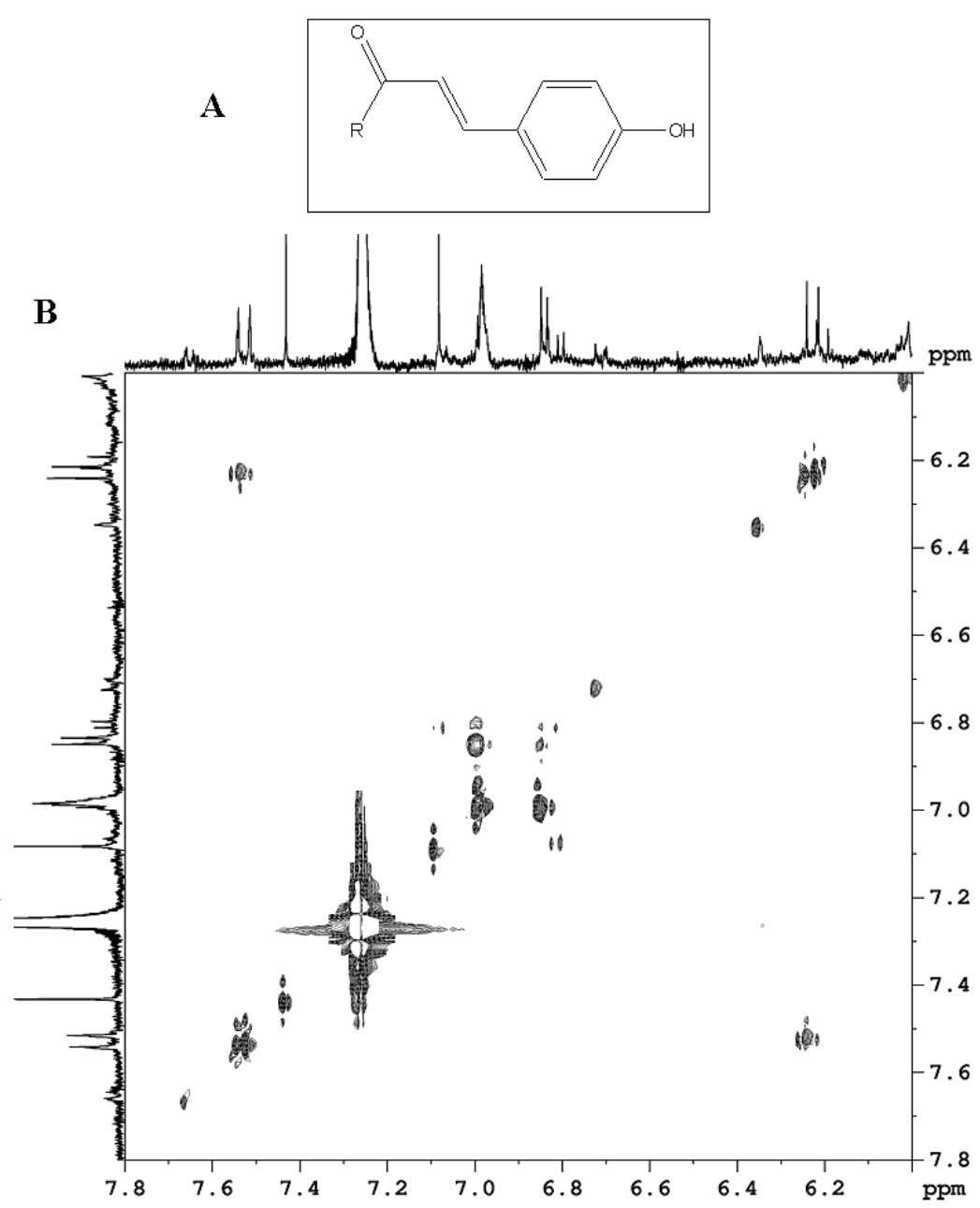

Figure 5. The chemical structure of para-cumaric acid derivative (A). The expansion 7.8-6.0 ppm of TOCSY map of rice oil (S8) (B). The horizontal and vertical projections are the corresponding region of ${ }^{1} \mathrm{H}$ NMR spectrum

\section{Conclusions}

The present study describes an analytical protocol useful to give consumers information on the quality of commercial edible oils. If we consider valid the quality criteria used for olive oil, the quality of commercial flavoured oils is poor. Only one sample was classified as "extra-virgin". The acidity value determined using traditional titration did not highlight the poor quality of these products whereas UV indices as well as $s n-1,2$ / $s n-1,3$ diglycerides ratio gave clear quality indication.

In the case of olive-sunflower oils only a minor fraction of olive oil was revealed.

Regarding the seed oils, some considerations can be made. Clearly, the aim of producers of cold pressed seed oils is to manufacture vegetable oils with better nutritional properties than those of the refined seed oils. These oils should derive only from organic cultivations avoiding chemical pesticides or so. On the other hand, using 
"organic cultivation", a risk factor for the possible presence of toxic contaminants (e.g.: aflatoxins) could be present. Some refinement processes not reported in the labels have been highlighted using Vis spectra. In a traditional refinement process, the neutralization step can be useful to eliminate aflatoxins that can be present in organic crops where pesticides are not used.

These results show that the quality of commercial products we analyzed is generally poor although they are commercialized at a very high price. In some cases, it is possible that the bottles were badly stored. However, the quality of a product should be assured at least until the expiration data. Moreover, in the case of mixtures of oils the percentage of the components should be reported as well as all the refinement treatment performed.

\section{Acknowledgements}

This work was carried as part of the research project of Sapienza University of Rome (Ateneo 2011, protocol C26A114A73) entitled: "Flavoured oils and seed oils with high nutritional value: analytical profile and evaluation of the quality by Nuclear Magnetic Resonance and conventional analyses". Authors thank Dr. Lorenzo Natale from Laboratorio Chimico Merceologico, Camera di Commercio dell'Industria, Agricultura e Artigianato of Rome, who performed GLC analyses of seed oils.

\section{References}

Angerer, P., \& von Schacky, C. (2000). n-3 polyunsaturated fatty acids and the cardiovascular system. Current opinion in lipidology, 11(1), 57-63. http://dx.doi.org/10.1097/00041433-200002000-00009

Antoun, N., \& Tsimidou, M. (1997). Gourmet olive oils: stability and consumer acceptability studies. Food Research International, 30, 131-136. http://dx.doi.org/10.1016/S0963-9969(97)00037-9

Belitz, H. D., Grosch, W., \& Schieberle, P. (2009). Food Chemistry. Berlin: Springer.

Bondioli, P., Bernardi, S., Mariani Costantini, A., Sala, M., \& Venturini, S. (1999). Oli di semi di spremitura. Sviluppi normativi e ricadute sulla tecnologia. Rivista Italiana Sostanze Grasse, 76, 1-9.

Braun, S., Kalinowski, H. O., \& Berger, S. (1998). 150 and More Basic NMR Experiments. Wiley-VCH: Weinheim.

Cabras, P., \& Martelli, A. (2004). Chimica degli alimenti. Piccin.

Carsten, F., Raniero, F., \& Guillou, C. (2000). ${ }^{1}$ H NMR as a tool for the analysis of mixtures of virgin olive oil with oils of different botanical origin. Magnetic Resonance in Chemistry, 38, 436-443. http://dx.doi.org/10.1002/1097-458X(200006)38:6<436::AID-MRC672>3.0.CO;2-X

D’Imperio, M., Gobbino, M., Picanza, A., Costanzo, S., Della Corte, A., \& Mannina, L. (2010). Influence of harvest method and period on olive oil composition: an NMR and statistical study. Journal of Agricultural and Food Chemistry, 58, 11043-11051. http://dx.doi.org/10.1021/jf1026982

Dwivedi, C., Natarajan, K., \& Matthees, D. P. (2005). Chemopreventive effects of dietary flaxseed oil on colon tumor development. Nutrition and Cancer, 51(1), 52-58. http://dx.doi.org/10.1207/s15327914nc5101_8

European Communities [EC]. (2003). Regulation No 1989/2003 amending Regulation (EEC) No 2568/91.

Greger, H., \& Hofer, O. (1980). New unsymmetrically substituted tetrahydrofurofuran lignans from artemisia absinthium: Assignment of the relative stereochemistry by lanthanide induced chemical shifts. Tetrahedron, 36(24), 3551-3558. http://dx.doi.org/10.1016/0040-4020(80)88051-3

Italian law, "Salari law", 27 January 1968- G.U. n.37 12/02/1968

Kang, S. S., Kim, J. S., Jung, J. H., \& Kim, Y. H. (1995). NMR Assignments of Two Furofuran Lignans from Sesame Seeds. Archives of Pharmacal Research, 18(5), 361-363. http://dx.doi.org/10.1007/BF02976333

Laggoune, S., Brouard, I., Leon, F., Calliste, C., Duroux, J., Bermejo, J., ... Kabouche, A. (2011). Lignans and an Abundant Flavone Glycoside with Free-Radical Scavenging Activity from the Roots of the Endemic Species Stachys mialhesi de Noé. Records of Natural Products, 5(3), 238-241.

Mannina, L., Luchinat, C., Emanuele, M. C., \& Segre, A. (1999). Acyl positional distribution of glycerol tri-esters in vegetable oils: a ${ }^{13} \mathrm{C}$ NMR study. Chemistry and Physics of Lipids, 103(1-2), 47-55. http://dx.doi.org/10.1016/S0009-3084(99)00092-4

Mannina, L., Luchinat, C., Patumi, M., Emanuele, M. C., Rossi, E., \& Segre A. (2000). Concentration dependence of ${ }^{13} \mathrm{C}$ NMR spectra of triglycerides: implications for the NMR analysis of olive oils. Magnetic $\begin{array}{llll}\text { Resonance } & \text { Chemistry, } & \text { 886-890. }\end{array}$ http://dx.doi.org/10.1002/1097-458X(200010)38:10<886::AID-MRC738>3.0.CO;2-J 
Mannina, L., Patumi, M., Proietti, N., Bassi, D., \& Segre, A. L. (2001). Geographical Characterization of Italian Extra Virgin Olive Oils Using High-Field ${ }^{1} \mathrm{H}$ NMR Spectroscopy. Journal of Agricultural and Food Chemistry, 49, 2687-2696. http://dx.doi.org/10.1021/jf001408i

Mannina, L., Sobolev, A. P., \& Segre, A. L. (2003). Olive oil as seen by NMR and chemometrics. Spectrosctropy Europe, 15(3), 6-14.

Mannina, L., \& Sobolev, A. P. (2011). High resolution NMR characterization of olive oils in terms of quality, authenticity, and geographical origin. Magnetic Resonance in Chemistry, 49, S3-S11. http://dx.doi.org/10.1002/mrc.2856

Mannina, L., D’Imperio, M., Gobbino, M., D’Amico, I., Casini, A., \& Sobolev, A. P. (2012). Nuclear magnetic resonance study of flavoured olive oils. Flavour and Fragrance Journal, 27, 250-259. http://dx.doi.org/10.1002/ffj.3094

Pérez-Camino, M. C., Moreda, W., \& Cert, A. (2001). Effects of Olive Fruit Quality and Oil Storage Practices on the Diacylglycerol Content of Virgin Olive Oils. Journal of Agricultural and Food Chemistry, 49, 699-704. http://dx.doi.org/10.1021/jf001064w

Raghuram, T. C., \& Rukimini, C. (1995). Nutritional significance of rice bran oil. Indian Journal of Medical Research, 102, 241-244.

Rangkadilok, N., Pholphana, N., Mahidol, C., Saengsooksree, W. W. K., Nookabkaew, S., \& Satayavivad, J. (2010). Variation of sesamin, sesamolin and tocopherols in sesame (Sesamum indicum L.) seeds and oil products in Thailand. Food Chemistry, 122, 724-730. http://dx.doi.org/10.1016/j.foodchem.2010.03.044

Segre, A. L., \& Mannina, L. (1997). ${ }^{1}$ H-NMR study of edible oils. Recent Research Developments in Oil Chemistry, 1, 297-308.

Silveira, M. B., Carraro, R., Monereo, S., \& Tébar, J. (2007). Conjugated linoleic acid (CLA) and obesity. Public Health Nutrition, 10(10A), 1181-1186. http://dx.doi.org/10.1017/S1368980007000687

Spyros, A., Philippidis, A., \& Dais, P. (2004). Kinetics of Diglyceride Formation and Isomerization in Virgin Olive Oils by Employing ${ }^{31} \mathrm{P}$ NMR Spectroscopy. Formulation of a Quantitative Measure to Assess Olive Oil Storage History. Journal of Agricultural and Food Chemistry, 52, 157-164. http://dx.doi.org/10.1021/jf030586j

Sugano, M., Koba, K., \& Tsuji, E. (1999). Health benefits of rice bran oil. Anticancer Research, 19, 3651-3657.

Thompson, L. U., Rickard, S. E., Orcheson, L. J., \& Seidl, M. M. (1996). Flaxseed and its lignan and oil components reduce mammary tumor growth at a late stage of carcinogenesis. Carcinogenesis, 17(6), 1373-1376. http://dx.doi.org/10.1093/carcin/17.6.1373

Vlahov, G. (2009). ${ }^{13} \mathrm{C}$ Nuclear Magnetic Resonance Spectroscopy to Determine Fatty Acid Distribution in Triacylglycerols of Vegetable Oils with "High - Low Oleic Acid" and "High Linolenic Acid". The Open Magnetic Resonance Journal, 2, 8-19. http://dx.doi.org/10.2174/1874769800902010008

Wang, Y. W., \& Jones, P. J. (2004). Conjugated linoleic acid and obesity control: efficacy and mechanisms. International Journal of Obesity, 28, 941-955. http://dx.doi.org/10.1038/sj.ijo.0802641

Warner, K., Vick, B., Kleingartner, L., Isaak, R., \& Doroff, K. (2003). Compositions of Sunflower, NuSun (Mid-Oleic Sunflower) and High-Oleic Sunflower Oils. In National Sunflower Association. Retrieved from http://www.sunflowernsa.com/uploads/resources/51/warner_.pdf

\section{Copyrights}

Copyright for this article is retained by the author(s), with first publication rights granted to the journal.

This is an open-access article distributed under the terms and conditions of the Creative Commons Attribution license (http://creativecommons.org/licenses/by/3.0/). 